\title{
Small, rare and little known: new records and species of Cardiomya (Bivalvia: Cuspidariidae) from Brazil
}

\author{
Tarcilla CARVALHO DE LIMA ${ }^{1, *}$, Cléo Dilnei de Castro OLIVEIRA² \& \\ Ricardo Silva ABSALÃO ${ }^{3}$ \\ 1,2,3 Universidade Federal do Rio de Janeiro, Instituto de Biologia, \\ Laboratório de Malacologia. C.E.P.: 21941-590. Rio de Janeiro, Brazil. \\ ${ }^{*}$ Corresponding author: tarcillacarvalho@gmail.com \\ ${ }^{2}$ Email: cleo.oliveira@gmail.com \\ 32Email: absalao@hotmail.com \\ ${ }^{1}$ urn:Isid:zoobank.org:author:7FFCF3E3-A9BF-469F-964A-62C618DE5192 \\ ${ }^{2}$ urn:lsid:zoobank.org:author:4BE74DFD-DD38-4315-A461-1E05F9706642 \\ ${ }^{3}$ urn:lsid:zoobank.org:author:D89BFB87-8BAF-44C4-AABD-5A40E202EE4C
}

\begin{abstract}
This paper describes rare Cardiomya species from Brazil which have been hitherto misidentified as Cardiomya cleryana (d'Orbigny, 1842) in literature or museum collections. Cardiomya minerva sp. nov. is proposed as new species and is characterized by its quadrangular shell, short and truncated rostrum, and external ornamentation composed of six radial ribs on the posterior half of the shell flank. Cardiomya striolata (Locard, 1897) described from the Mediterranean Sea and northwestern Atlantic Ocean, is reported from Brazil for the first time; although previously regarded as a junior synonym of Cardiomya costellata (Deshayes, 1835), it is herein considered as a full species and redescribed. This species is characterized by its trapezoidal shell flank, elongated rostrum, tapering towards the tip, and external ornamentation composed of 18-53 radial ribs, the 3-4 posterior ones being the strongest and more widely spaced. Other three previously unknown species are illustrated but not formally named due to the lack of well-preserved articulated shells.
\end{abstract}

Keywords. Mollusca, Septibranchia, biodiversity, taxonomy.

Carvalho de Lima T., Oliverira C.D. de C. \& Absalão R.S. 2020. Small, rare and little known: new records and species of Cardiomya (Bivalvia: Cuspidariidae) from Brazil. European Journal of Taxonomy 619: 1-20. https://doi.org/10.5852/ejt.2020.619

\section{Introduction}

Cuspidariidae Dall, 1886 is a family of marine carnivorous bivalves (Allen \& Morgan 1981; Morton 1981). Most species are less than $10 \mathrm{~mm}$ in length, and many of them are rare, only known by their original descriptions and few subsequent records. This rarity plus the fragility of the cuspidariid shells led to the scarcity of available specimens in scientific collections, where most species are represented only by few and usually broken shells. 
Due to the numerous misidentifications in literature and in museum collections, many species are still not described or even recognized as new to science. A more complex problem of identification appears in Cardiomya A. Adams, 1864, where species are very similar, most of them with shells displaying few and variable characters.

Four species of Cardiomya have been reported from Brazil (Rios 2009; Oliveira et al. 2017): Cardiomya cleryana (d'Orbigny, 1842), C. ornatissima (d'Orbigny, 1853), C. striata (Jeffreys, 1876) and C. perrostrata (Dall, 1881).

Nevertheless, there are rare and little known Cardiomya still hidden on museums' shelves. Remarkable, most of them have been lumped with C. cleryana. The present study aims at reviewing these species.

\section{Material and methods}

We examined all available Cardiomya material from the Atlantic ocean found in the following institutions:

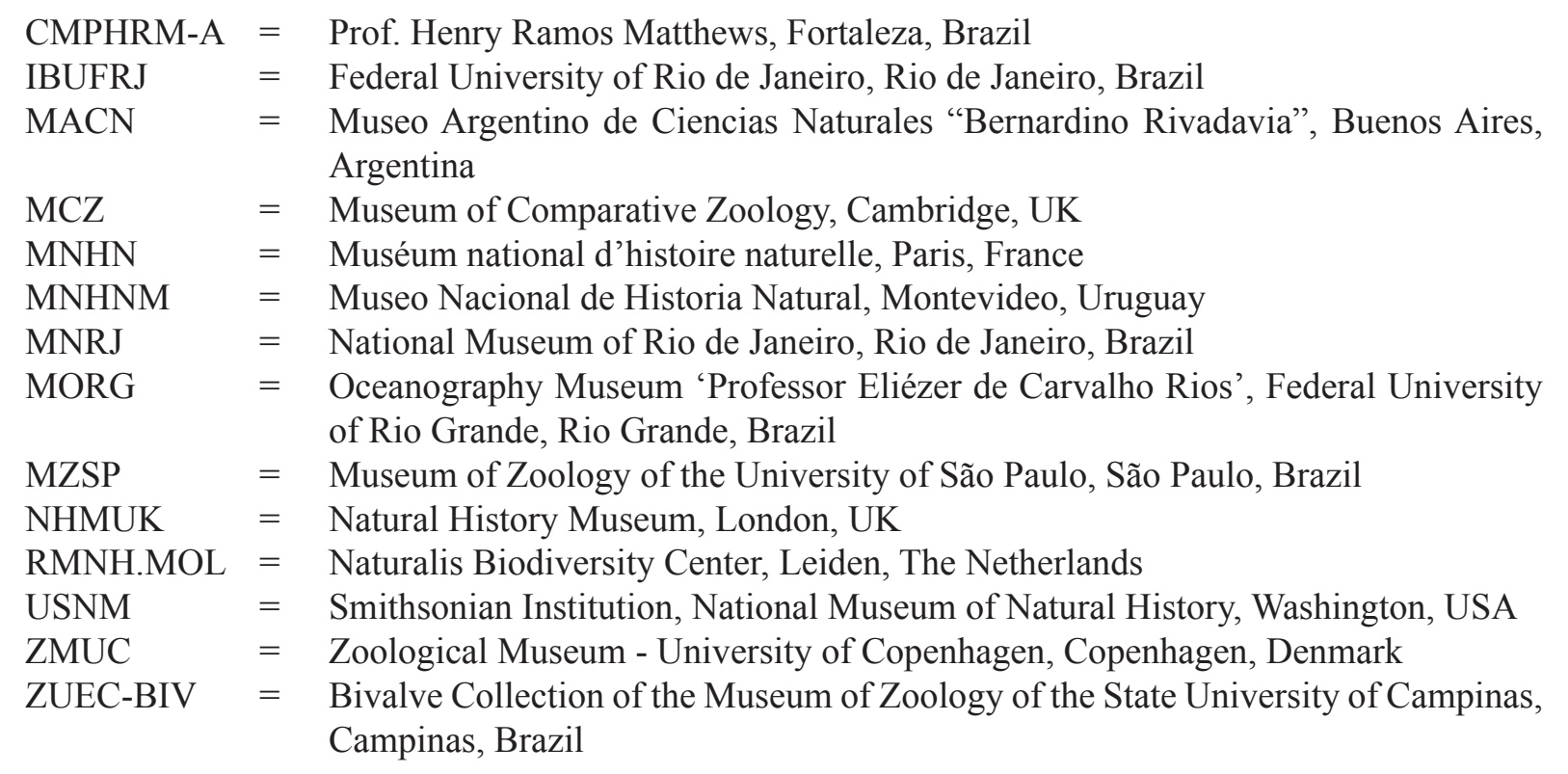

Specimens were compared with the type material and/or original descriptions. For the unknown species, only that with well preserved and articulated specimens are proposed as new.

Literature records were critically assessed and an updated geographical distribution of the species considered is provided. Records originating from checklists, unvouchered material, and non figured or properly described specimens are not included. Each species is compared with the most similar species or to those species with which they have been confused in the literature and/or in malacological collections. A pictogram illustrates the terminology used for the shell structures (Fig. 1). Higher taxonomy follows Gofas (2015). Species descriptions are based on original description and type/ordinary material examined. Table 1 summarizes the characters of the Cardiomya species studied here.

Only the lots containing types or ordinary material important for the discussions are listed. The preservation status of specimens is coded as ' $\mathrm{s}$ ' for articulated valves without soft parts (shells), ' $\mathrm{v}$ ' for single valves, and 'i' for complete individuals; i.e., shells containing soft parts. 


\title{
Results
}

\section{Taxonomy}

\author{
Phylum Mollusca Linnaeus, 1758 \\ Class Bivalvia Linnaeus, 1758 \\ Superorder Anomalodesmata Dall, 1889 \\ Superfamily Cuspidarioidea Dall, 1886 \\ Family Cuspidariidae Dall, 1886 \\ Genus Cardiomya A. Adams, 1864
}

Type-species (by monotypy)

Neaera gouldiana Hinds, 1843: 77.

\section{Description}

Inequivalve, left valve slightly overlapping right valve along ventral margin, right valve overlapping left valve on dorsal margin of rostrum. External sculpture of radial ribs and commarginal growth lines. Hinge: left valve edentulous, right valve with a strong posterior lateral tooth. Ligament internal (Allen \& Morgan 1981; Poutiers \& Bernard 1995; Barroso et al. 2016; Machado et al. 2016; Oliveira et al. 2017).
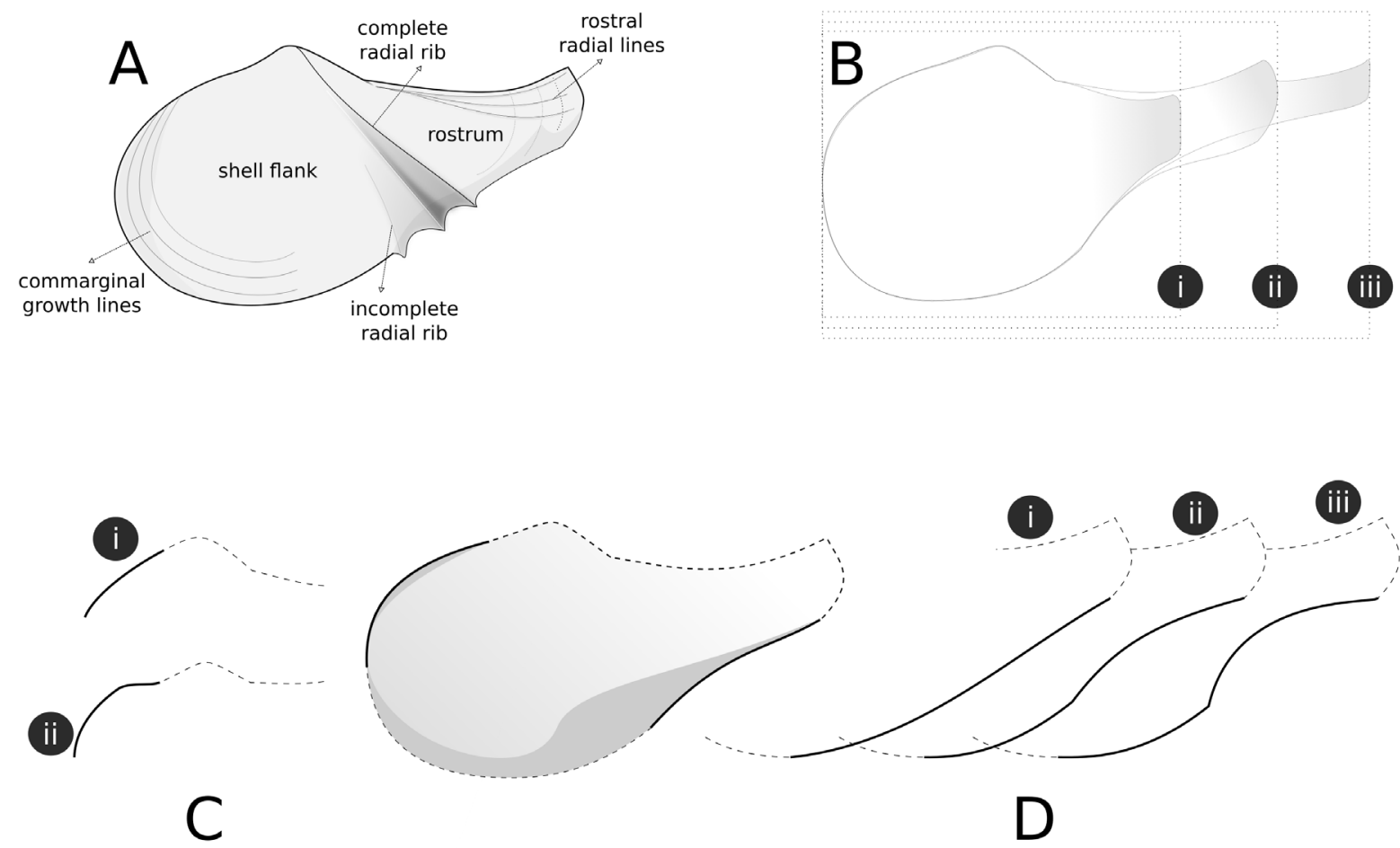

Fig. 1. Pictographic glossary. A. General shell features. B. Rostrum length, termed as very short (Bi), short (Bii) and long (Biii). C. Anterodorsal margin, termed as descending (Ci) and forming a shoulder (Cii). D. Posteroventral sinuation, termed as unobtrusive (Di), shallow (Dii) and gently marked (Diii). 


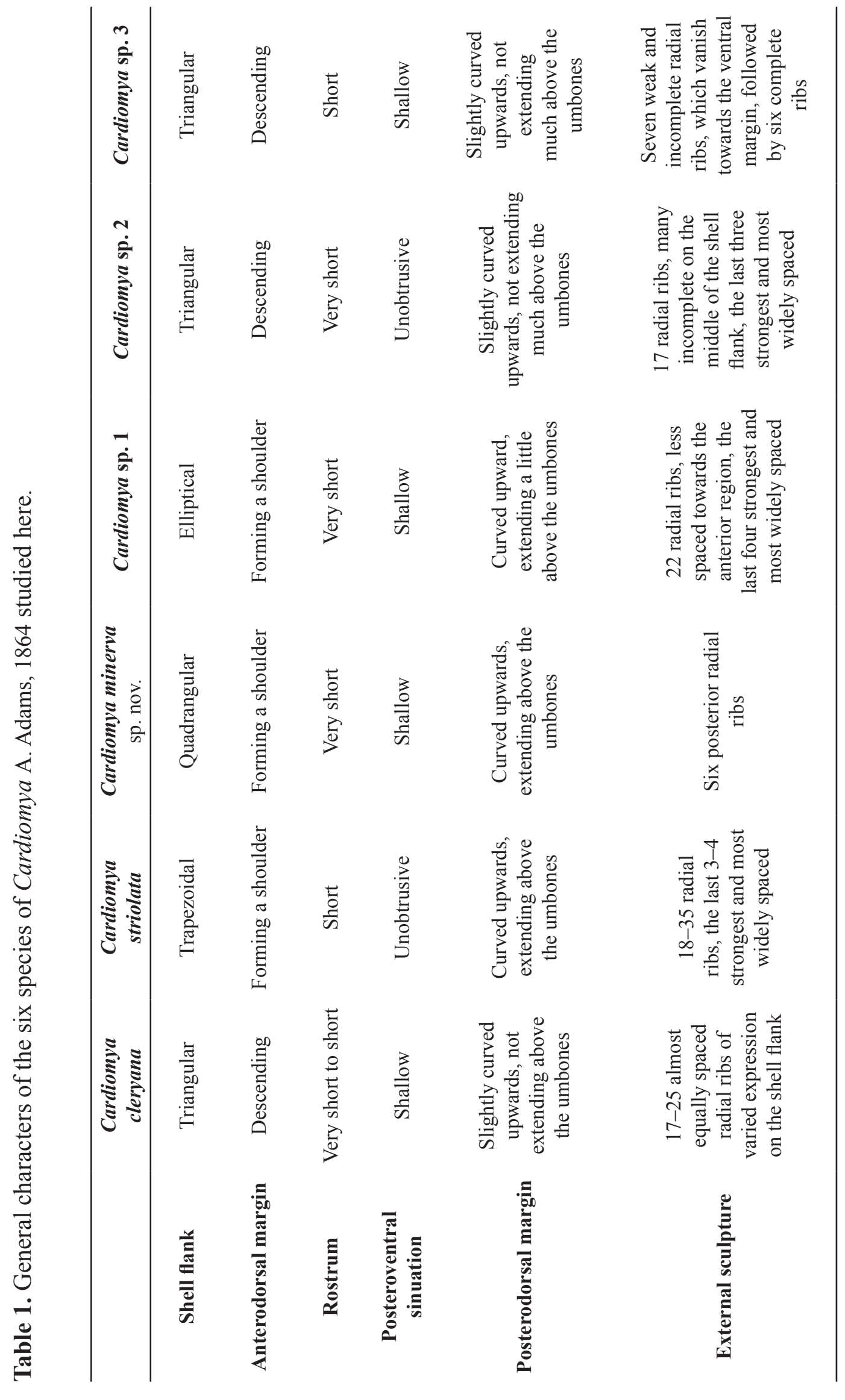


Cardiomya cleryana (d'Orbigny, 1842)

Fig. 2A-E

Sphenia cleryana d'Orbigny, 1842: 572 (description, as "Sphena Cleryana"), 708 (reference to illustrations, as "Sphaenia Cleryana"); 1846: pl. 83, figs 16-18 (as "Sphaenia Cleryana").

Cuspidaria (Cardiomya) simillima E.A. Smith, 1915: 104, pl. 2, fig. 24.

Sphenia cleryana - d'Orbigny 1853: 285.

Cuspidaria cleryana - Morretes 1949: 52.

Cardiomya cleryana - Rios 1970: 219; 1975: 261, pl. 85, fig. 1255; 1985: 281, pl. 99, fig. 1385; 1994: 303, pl. 104, fig. 1483; 2009: 606, fig. 1678. — Figueiras \& Sicardi 1980: 157. — Layerle \& Scarabino 1984: 6. - Poutiers \& Bernard 1995: 151, 158. — Forcelli 2000: 171, figured. Scarabino 2003a: 238. — Absalão et al. 2006: 223, 258. — Allen 2011: 440. — Passos \& Magalhães 2011: 7. - Oliveira \& Sartori 2014: 10, 13-14, fig. 2D-E. - Barroso et al. 2016: 2, 6-8, 11, in part. — Machado et al. 2016: in part. - Oliveira et al. 2017: 2, 4-6, 8-9, 11, 14, in part.

Cuspidaria (Cardiomya) simillima - Powell 1951: 51. — Figueiras \& Sicardi 1970: 420-421, pl. vi, fig. 93. - Poutiers \& Bernard 1995: 166. — Oliveira et al. 2017: 289, fig. 3C-D.

Cuspidaria simillima - Castellanos 1982: 41.

Cardiomya simillima - Rios 1970: 220; 1972: 197. —Zelaya 2005: 117.

Cardiomya perrostrata auct., non Dall, 1881 - Rios 1975: 261, pl. 85, fig. 1257; 1985: 281, pl. 99, fig. 1387; 1994: 303, pl. 104, fig. 1485. — Absalão \& Pimenta 2005: 51, fig. 134.

\section{Material examined}

Type material

BRAZIL • [4v], syntypes of Cardiomya simillima (Smith, 1915); off Rio de Janeiro; $73 \mathrm{~m}$ depth; NHMUK 1915.4.18.512-515.

CARIBBEAN SEA - [4v and fragments], syntypes of Cardiomya ornatissima (d'Orbigny, 1853); NHMUK 1854.10.4.554.

COUNTRY UNKNOWN • [fragments], syntypes of Cardiomya striata (Jeffreys, 1876); North Atlantic; 1260-2651 m depth; NHMUK 1877.11.28.50.

DENMARK • [2v and fragments], syntypes of Cardiomya striata; Greenland; $56^{\circ} 11^{\prime} 00^{\prime \prime} \mathrm{N}, 37^{\circ} 41^{\prime} 00^{\prime \prime} \mathrm{W}$; 2652 m depth; Valorous Expedition 1875, stn 12; USNM 63965 - [fragments], syntypes of Cardiomya striata; Greenland; 56 $06^{\prime} 00^{\prime \prime} \mathrm{N}, 34^{\circ} 42^{\prime} 00^{\prime \prime} \mathrm{W}$; $1262 \mathrm{~m}$ depth; Valorous Expedition 1875, stn 13; USNM 172288 .

IRELAND • [1s], syntype of Cardiomya striata; Rockall Bank; 56 $07^{\prime} 00^{\prime \prime} \mathrm{N}, 14^{\circ} 19^{\prime} 00^{\prime \prime} \mathrm{W} ; 1152 \mathrm{~m}$ depth; Porcupine Expedition 1869, stn 23; USNM 172289 • [fragments], syntypes of Cardiomya striata; Rockall Bank; 56 $13^{\prime} 00^{\prime \prime} \mathrm{N}, 14^{\circ} 18^{\prime} 00^{\prime \prime} \mathrm{W} ; 768 \mathrm{~m}$ depth; Porcupine Expedition 1869, stn 23-A; USNM 172290.

SCOTLAND • [1s], syntype of Cardiomya striata; Off Hebrides islands; $60^{\circ} 45^{\prime} 00^{\prime \prime}$ N, $04^{\circ} 49^{\prime} 00^{\prime \prime}$ W; 933 m depth; Lightning Expedition 1868, stn 6; USNM 172286 • [1s], syntype of Cardiomya striata; Off Hebrides islands, $59^{\circ} 36^{\prime} 00^{\prime \prime} \mathrm{N}, 07^{\circ} 20^{\prime} 00^{\prime \prime} \mathrm{W}$; $969 \mathrm{~m}$ depth; Lightning Expedition 1868, stn 12; USNM 172287.

UNITED STATES - Florida state - [2s], syntypes of Cardiomya perrostrata (Dall, 1881); off Dry Tortugas; $24^{\circ} 08^{\prime} 00^{\prime \prime} \mathrm{N}, 82^{\circ} 51^{\prime} 00^{\prime \prime} \mathrm{W}$; $620 \mathrm{~m}$ depth; Blake Expedition, stn 43; USNM $63119 \bullet$ [1s], syntype of Cardiomya perrostrata; same collecting data as for preceding; MCZ 8100. 
Other material

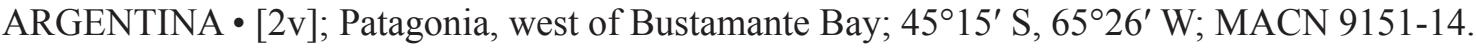

BRAZIL - Pernambuco state • [in part, 5v]; Ilha de Itamaracá; CMPHRM 3659. - Espírito Santo state • [in part, 2v]; Guarapari; MZSP 77301. - Rio de Janeiro state • [4v]; 107 m depth; USNM 96108 - [2v]; 06 Aug. 1979; IBUFRJ 3355 - [in part, 1v]; Macaé, APA de Santana; 5-10 m depth; IBUFRJ 6434. - São Paulo state - [in part, 2v]; Caraguatatuba; $23^{\circ} 44^{\prime} 40^{\prime \prime} \mathrm{S}, 45^{\circ} 01^{\prime} 14^{\prime \prime} \mathrm{W} ; 40 \mathrm{~m}$ depth; 16 Oct. 2001; ZUEC-BIV 2217 • [in part, 2v]; Caraguatatuba; $23^{\circ} 44^{\prime} 16^{\prime \prime} \mathrm{S}, 45^{\circ} 03^{\prime} 02^{\prime \prime} \mathrm{W} ; 45 \mathrm{~m}$ depth; 16 Oct. 2001; ZUEC-BIV 2218 • [2v]; São Sebastião, Praia do Araçá; 2349'20" S, 45²4'10" W; 21 Mar. 2014; ZUEC-BIV 5135 • [19v]; São Sebastião; 29 Apr. 1949; MNHNM 3281 • [4v]; Ubatuba, Praia da Enseada; 10 m depth; 25 May 1967; MZSP 57255 • [1s, 5v]; Tabatinga, Praia de Mococa; MZSP 87193. - Rio Grande do Sul state - [20v]; Off Rio Grande; 100 m depth; 4 Apr. 1998; Revizee, stn 6839; MORG 50583.

UNITED STATES - Florida state • [1s, 2v]; off West'n Dry Rocks; 164 m depth; 1916; USNM 460734.

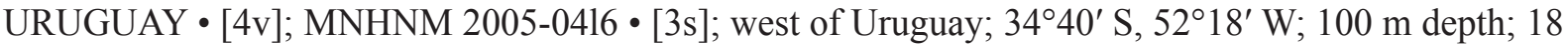
Sep. 1938; MACN 23465.

\section{Description}

Shell small (maximum length $10.5 \mathrm{~mm}$; maximum height $8 \mathrm{~mm}$ ). Shell flank triangular, umbones blunt. Anterodorsal margin descending, anterior margin rounded little extended, ventral margin rounded, crenulated. Rostrum very short to short, posteroventral sinuation shallow, posterodorsal margin slightly curved upwards, not extending above the umbones. External sculpture of 17-25 almost equally

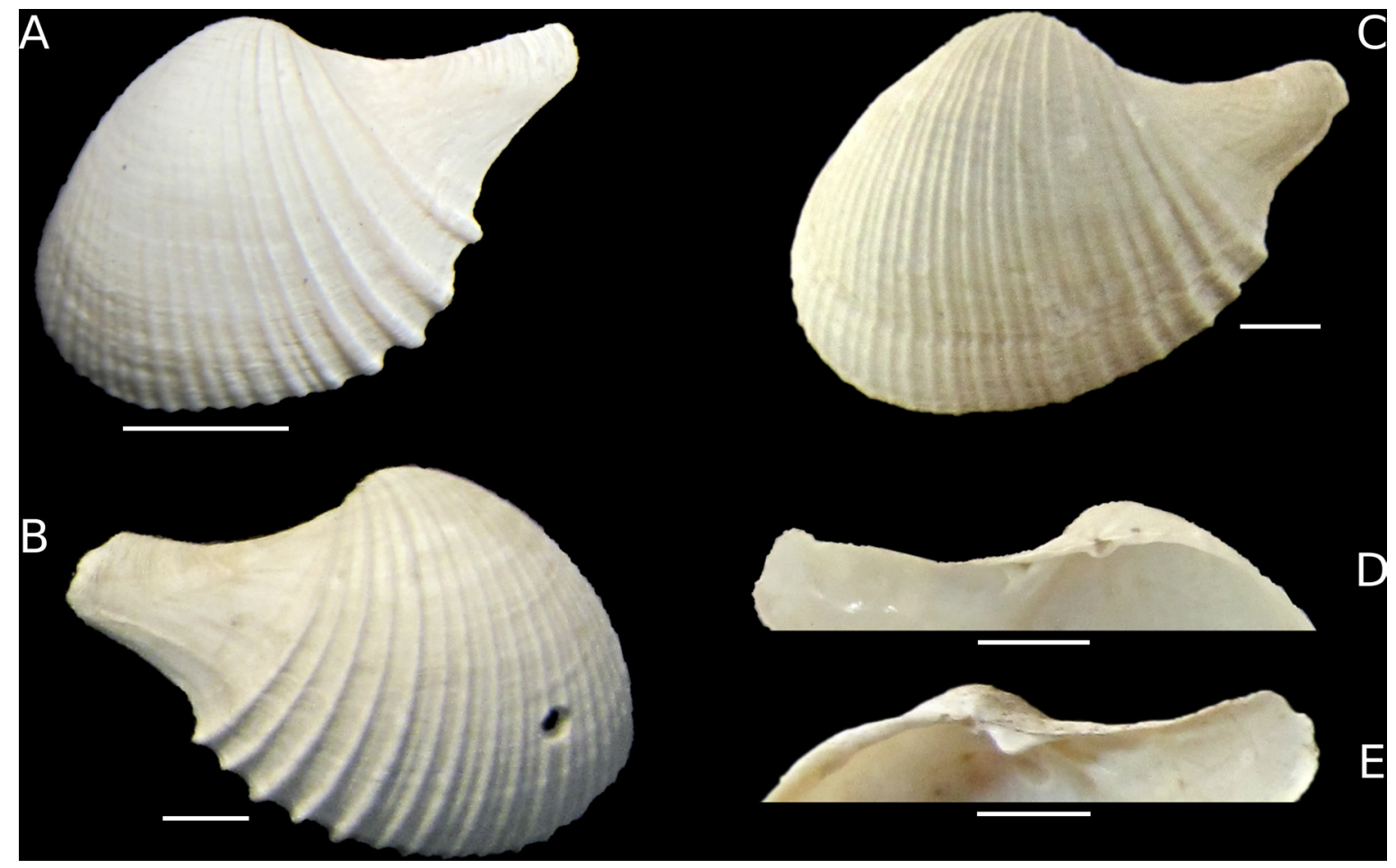

Fig. 2. Cardiomya cleryana (d'Orbigny, 1842). A-C. External view. A. MNHNM 3281, left valve. B. USNM 96108, right valve. C. MORG 50583, left valve. D-E. MORG 50583, hinge detail. Scale bars $=2 \mathrm{~mm}$. 
spaced radial ribs of varied expression on the shell flank; rostrum with one to three radial lines or only commarginal growth lines.

\section{Type localities}

Jamaica, Cuba, Guadeloupe, Saint Thomas and Brazil, Cape São Tomé, Rio de Janeiro, 80 m.

\section{Geographic distribution}

Florida, USA to Chubut Province, Argentina. Confirmed records: United States: Florida, Off West's Dry Rocks (USNM 460734). Brazil: East and Northeast (Rios 1970), Pernambuco state: Ilha de Itamaracá (CMPHRM 3659); Espírito Santo state: Guarapari (MZSP 77301); Rio de Janeiro State: Macaé, APA de Santana (IBUFRJ 6434), Rasa Island (Rios 1970), off Juatinga, Paraty (Rios 1970); São Paulo State: Mocóca (Morretes 1949), Tabatinga (MZSP 87193), Ubatuba (MZSP 57255), Caraguatatuba (ZUECBIV 2217-2218), São Sebastião (Morretes 1949); Rio Grande do Sul State: Southeast of Rio Grande (Rios 1972), off Torres, Tramandaí, Solidão, Conceição, Cassino, Sarita, Albardão and Chuí (Rios 1975). Uruguay: 100 miles south of Rocha Department (Figueiras \& Sicardi 1970). Argentina: Chubut Province: west of Cruz Bay (Castellanos 1982); Patagonia (Zelaya 2005). Bathymetry: from $49 \mathrm{~m}$ (Rios 1970) to $180 \mathrm{~m}$ (Rios 1972).

\section{Remarks}

Cardiomya ornatissima differs from C. cleryana by its straight posterodorsal margin by having only and 6-7 strong radial ribs, which may be followed or not by incomplete ribs. Cardiomya perrostrata can be distinguished from C. cleryana by its long rostrum, rounded shell flank, and the presence of a shoulder. Cardiomya striata can be distinguished from C. cleryana by its quadrangular shell flank, well marked shoulder, and radial ribs covering the entire shell, including the rostrum.

Cardiomya simillima, described from Rio de Janeiro (73 m depth) and Malvinas/Falkland Islands (229 m depth), exhibits the same shell outline and ornamentation than C. cleryana and it was therefore usually considered as a junior synonym (Rios 1975, 2009; Poutiers \& Bernard 1995; Forcelli 2000; Scarabino 2003b; Bouchet 2009; Barroso et al. 2016; Machado et al. 2016; Oliveira et al. 2017), an opinion with which we agree.

The record of $C$. simillima to Malvinas/Falkland Islands was uncritically replicated by many authors (e.g., Powell 1951; Figueiras \& Sicardi 1970; Rios 2009; Barroso et al. 2016; Machado et al. 2016). Nevertheless, subsequent records from Malvinas/Falkland Islands were never provided. As has happened to others species records from the Terra Nova expedition (for details see Scarabino 2003b; Signorelli et al. 2019), it is possible that a labeling error occurred in the record of C. simillima from station \#38 (Malvinas/Falkland Islands, $229 \mathrm{~m}$ ). Taking into account the evidence of tag scrambling and also the biogeographical implications of such an unusual distribution, the record of station \#38 from Terra Nova expedition to $C$. simillima $(=C$. cleryana) should be viewed with caution.

Cardiomya striolata (Locard, 1897)

Figs $3 \mathrm{~A}-\mathrm{F}, 4 \mathrm{~A}-\mathrm{H}$

Cuspidaria striolata Locard, 1897: 94-95.

Cuspidaria striolata - Locard 1898: 195-196, pl. VIII, figs 20-25; 1899: 130. — Poutiers \& Bernard 1995: 151, 167. — Allen 2011: 441. 
Cardiomya costellata auct., non Deshayes, 1835 -Jeffreys 1865: 49-50; 1869: pl. XLIX, fig. 3. - Allen \& Morgan 1981: 464-466, fig. 29. - Salas 1996: 76, figs 137-138. — Porcheddu et al. 1997: 157-162, figs 1-3. - Mikkelsen \& Bieler 2008: 224, figured. — Tunnell et al. 2010: 339, figured. - Oliver et al. 2016.

Cardiomya cleryana auct., non d'Orbigny, 1842 - Barroso et al. 2016: 6-7, fig. 5. - Oliveira et al. 2017: 286, 291.

Cardiomya perrostrata auct., non Dall, 1881 - Barroso et al. 2016: 10, fig. 7A-B. - Machado et al. 2016: 3, figs 1A-B.

Cardiomya striata auct., non Jeffreys, 1876 - Oliveira et al. 2017: 284.

Cardiomya sp. 3 - Lamy \& Pointier 2018: 752, pl. 252, fig. 7.

Cardiomya sp. 7 - Lamy \& Pointier 2018: 755, pl. 252, fig. 11.

\section{Material examined}

Type material

SPAIN • [2v], syntypes of Cardiomya striolata (Locard, 1897); north of Spain, Bay of Biscay; $44^{\circ} 05^{\prime} \mathrm{N}$, 05³6' W; 608 m depth; Travailleur Expedition 1882, stn 02; MNHN 24210.

\section{Other material}

BARBADOS • [3s]; off Paynés Bay; 64-137 m depth; 1918; USNM 502902.

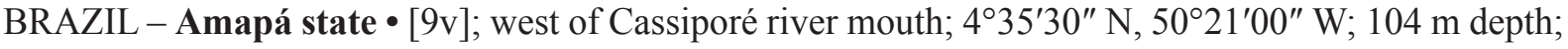
18 May 1971; GEOMAR III, stn 210; CMPHRM 3678 • [4v]; west of Amazon river mouth; 02 $01^{\prime} 00^{\prime \prime} \mathrm{N}$, 4732'30" W; 86 m depth; 20 Apr. 1971; GEOMAR III, stn 150; CMPHRM 3679 • [1v]; Amazon canyons; 02 $53^{\prime} 00^{\prime \prime} \mathrm{N}, 4^{\circ} 17^{\prime} 00^{\prime \prime} \mathrm{W}$; $112 \mathrm{~m}$ depth; 14 Sep. 1970; GEOMAR II, stn 129; CMPHRM

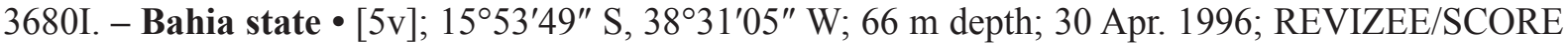
Central, stn C76; MNRJ 15892 • [5v]; Royal Charlotte Bank; 1553'49" S, 38³1'05" W; 66 m depth; 30 Apr. 1996; REVIZEE/SCORE Central, stn 76C; IBUFRJ 12337. - Espírito Santo state • [1i, 2v]; Trindade island; 20²9' S, 29¹8' W; 63 m depth; 22 May 1987; Marion Dufresne MD55, stn DC61; MNRJ 25957 • [1i]; Vitória-Trindade Seamounts; 2041'41" S, 34³5'02" W; 108 m depth; 12 Jul. 2001; REVIZEE/SCORE Central, stn 45R; IBUFRJ 21296. - Ceará state • [1v]; Canopus Bank; 0313' S, 38 31' W; CMPHRM 712A. - Pernambuco state • [2v]; Itamaracá island; CMPHRM 3695A. - Alagoas state • [1v]; Maceió; 09²7'08" S, 3507'07" W; 08 Sep. 1965; CMPHRM 3651A.

COUNTRY UNKNOWN • [7v]; Mediterranean; $82 \mathrm{~m}$ depth; 1870; USNM 172020 • [2s, 5v]; Mediterranean, off Soloom Bay; 73-220 m depth; USNM 172017 • [3v]; Aegean Sea; $450 \mathrm{~m}$ depth; USNM 172025.

IBERIAN PENINSULA • [2v]; Gibraltar; 1942; C.B. Adams leg.; MCZ 154438.

IRELAND • [In part, 10s and 2v]; northwest off Ireland; $300 \mathrm{~m}$ depth; 1869; Porcupine Expedition, stn 25; USNM 172007.

ITALY • [4v]; Naples; 11 Sep. 1937; S. Putzep leg.; MCZ 231146.

SCOTLAND • [1v]; Loch Fyne; MCZ 243626.

SPAIN • [2v]; Cartagena Bay; 84-153 m depth; 1870; USNM 172018.

\section{Description}

Shell small (maximum length $9.3 \mathrm{~mm}$; maximum height $6.3 \mathrm{~mm}$ ). Shell flank trapezoidal, umbones blunt. Anterodorsal margin forming a shoulder, anterior margin little extended, ventral margin rounded, 
crenulated. Rostrum short, tapering towards the tip, posteroventral sinuation unobtrusive, posterodorsal margin curved upwards, extending above the umbones. External sculpture of 18-35 radial ribs, weak and closely spaced anteriorly; becoming stronger posteriorly, the last three to four being the strongest and most widely spaced, the posterior most rib separated from the previous ones; commarginal growth lines well marked; rostrum with one to three radial lines.

\section{Type localities}

North of Spain, Bay of Biscay (608-1037 m depth). Southwest of Portugal, Gulf of Cádiz (2100 m depth). Mediterranean Sea, off Xauen Bank (322 m depth).

\section{Geographic distribution}

Mediterranean Sea, north Atlantic Ocean and Brazil. Confirmed records: United States: North Carolina to Florida (Mikkelsen \& Bieler 2008), Texas (Tunnell et al. 2010). West Indies (Mikkelsen \& Bieler 2008); Bahamas (Mikkelsen \& Bieler 2008); Barbados, off Paynés Bay (USNM 502902); Guadeloupe (Lamy \& Pointier 2018). Caribbean Central American (Mikkelsen \& Bieler 2008). Scotland: Shetland: Whales Skerries (Jeffreys 1865), Loch Fyne (MCZ 243626). Western Europe: Hebrides Seamount (Allen \& Morgan 1981). Dublin: off Ireland (USNM 172007). Italy: Sea of Sardinia, Tavolara island (Porcheddu et al. 1997), Naples (MCZ 231146). France: off Nantes (Allen \& Morgan 1981); west of

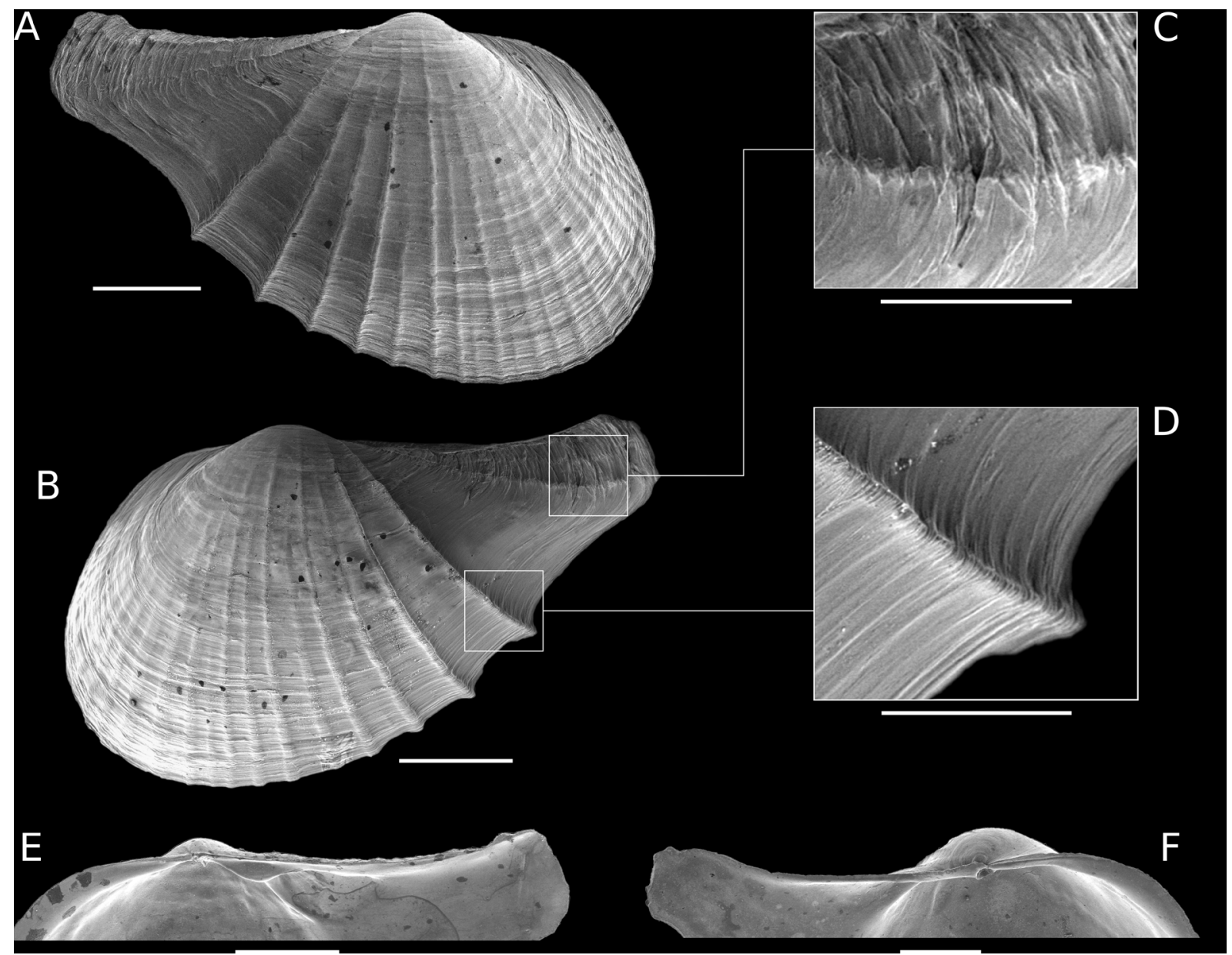

Fig. 3. Cardiomya striolata (Locard, 1897). A-E. IBUFRJ 21296. A-B. External view, right and left valve. C-D. Details of the rostrum and posterior radial rib. E. Hinge detail, right valve. F. CMPHRM 3679. Hinge detail, left valve. Scale bars: A-B, E-F $=1 \mathrm{~mm}$; $-\mathrm{D}=0.5 \mathrm{~mm}$. 
Brest (Allen \& Morgan 1981); Saint-Raphael (Locard 1898); Provence (Locard 1898); Marseille (Locard 1898); Bay of Biscay (Locard 1899; Allen \& Morgan 1981). Iberian Peninsula: Gibraltar (MCZ 154438). Spain: Cartagena Bay (USNM 172018). Gulf of Cádiz (type locality). Portugal: Cape St Vicent (Salas 1996); Off Huelva (Salas 1996); West of the Straith of Gibraltar (Salas 1996). Morocco: off Casablanca (Salas 1996); off Rabat (Salas 1996); East of Ceuta (Salas 1996); Alboran Ridge (Salas 1996); Xauen Bank (Salas 1996); West of Alboran basin (Salas 1996); Aegean sea: (USNM 172025). Mediterranean: (Poutiers \& Bernard 1995) (USNM 172020); off Soloom Bay (USNM 172017).Brazil: Amapá state, Amazon Canyons (CMPHRM 3680I); west of Cassiporé river mouth (CMPHRM 3679; CMPHRM 3678); Ceará state, Canopus, off Ceará (712A); Pernambuco state, Itamaracá Island (CMPHRM 3695A); Bahia state: (MNRJ 15892); Royal Charlotte Bank (IBUFRJ 12337); Espírito Santo state (MNRJ 25957). Bathymetry: from $50 \mathrm{~m}$ (Porcheddu et al. 1997) to $2100 \mathrm{~m}$ (Locard 1898).

\section{Remarks}

Cardiomya striolata (Locard, 1897), was usually regarded as a junior-synonym of Cardiomya costellata (Deshayes, 1835), with more strongly ribbed ornamentation (see Dall 1886; Salas 1996; Porcheddu et al. 1997). However, in our opinion C. striolata is a distinct, full species, which differs from C. costellata by its tapering rostrum which extends above the umbones, trapezoidal shell flank, anterodorsal margin forming a shoulder, and unobtrusive posteroventral sinuation.

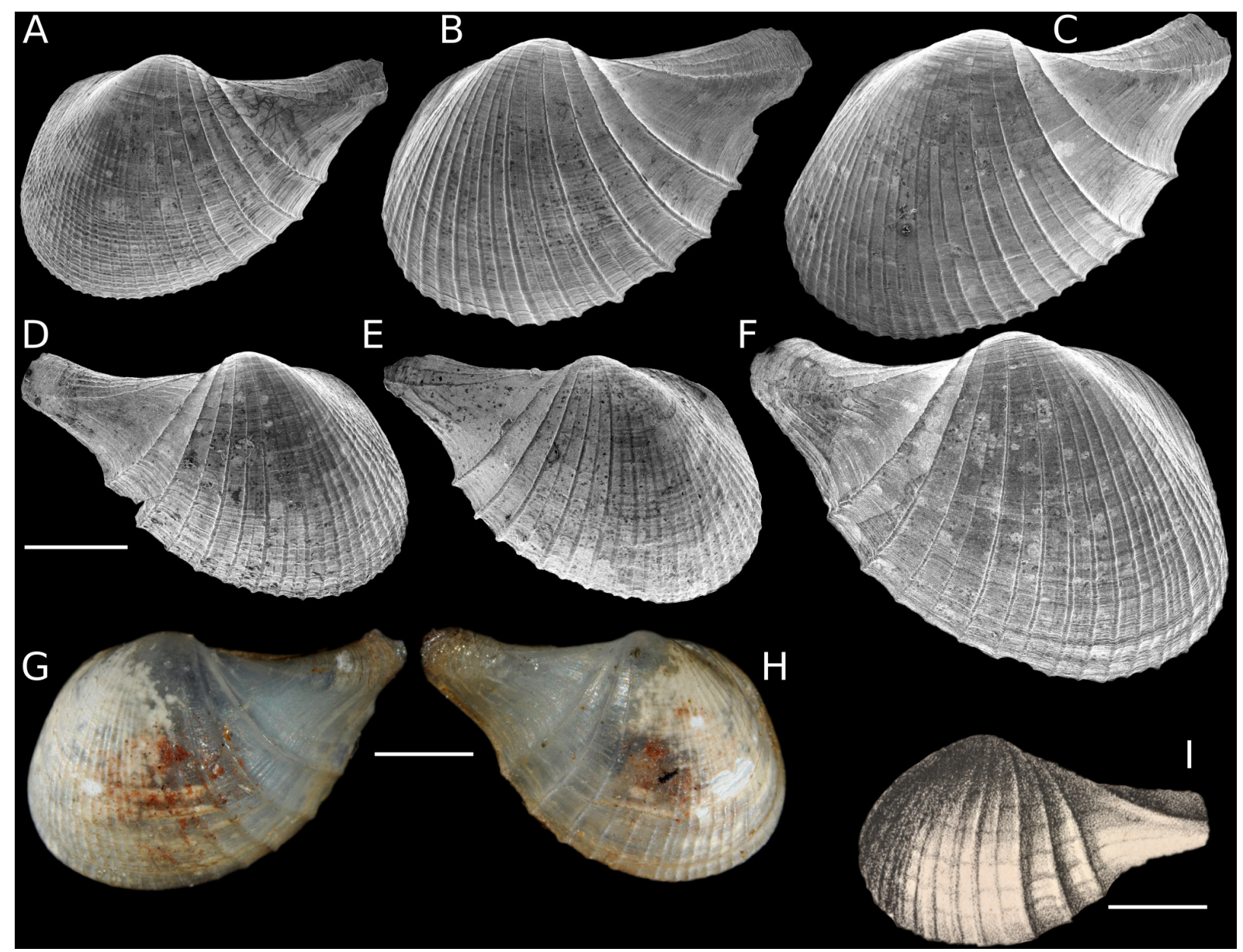

Fig. 4. A-H. Cardiomya striolata (Locard, 1897). External view. A-C. Left valve. D-F. Right valve. A, E. CMPHRM 3679. B-C, F. CMPHRM 3678. D. CMPHRM 3680I. G-H. Syntype MNHN 24210. - Cardiomya costellata (Deshayes, 1835). I. Original picture. Scale bars $=2 \mathrm{~mm}$. 
Cardiomya costellata was described based on fossil specimens from the Tertiary of Greece (Fig. 4I). Since its original description, several incorrect records of C. costellata have been made in a series of erroneous taxonomic identifications of different species (e.g., Philippi 1844; Abbott 1974; Abbott \& Morris 1995; Redfern 2001; Ardovini \& Cossignani 2004; Lee 2009; Morton 2016; Oliver et al. 2016; Lamy \& Pointier 2018). Literature records of true specimens of C. striolata could be confirmed in references of $C$. costellata accompanied by figures, detailed descriptions or cross-referenced with vouchers in collections. Additionally, many specimens of $C$. striolata labeled as C. costellata were found among museums holdings.

Considering the specimens from Brazil, Cardiomy a striolata has often been misidentified in the literature: [1] part of the specimens listed as C. cleryana in Barroso et al. (2016: 6), as well as the figured specimen in Barroso et al. (2016: 7, fig. 5). [2] the specimen figured in Machado et al. (2016: 3, figs 1A-B) as C. perrostrata and then identified as C. cleryana by Oliveira et al. (2017: 286). [3] the specimen from CMPHRM 712A reported upon as $C$. perrostrata by Barroso et al. (2016) and later identified as $C$. striata by Oliveira et al. (2017). [4] Absalão et al. (2006) recorded the occurrence of $C$. cleryana and $C$. perrostrata at Bahia State. These specimens were later identified as Cardiomya cleryana (MNRJ 15892) and a mix of C. cleryana and C. ornatissima (IBUFRJ 12337) by Oliveira et al. (2017: 291). However, the specimens identified as C. cleryana on both lots are actually Cardiomya striolata. The specimens of C. ornatissima were renumbered as IBUFRJ 21305.

Cardiomya striata can be distinguished from C. striolata by its rectangular shell flank and entire shell (including the rostrum) ornamented with radial ribs.

Cardiomya minerva sp. nov.

urn:1sid:zoobank.org:act:B7AF183B-CE39-4084-A9B1-E0F366458513

Fig. 5A-F

\section{Diagnosis}

Quadrangular shell flank, very short truncated rostrum, posterodorsal margin curved upwards, extending above the umbones; external sculpture composed of six posterior radial ribs, rostrum with commarginal growth lines only.

\section{Etymology}

This species is named after the character Minerva McGonagall, from the Harry Potter series of books written by the British J.K. Rowling. The name is employed as a noun in apposition.

\section{Material examined}

Holotype

BRAZIL - Alagoas state • [1i]; Maceió, Praia da Avenida; 10 m depth; 10 Apr. 1990; IBUFRJ 5785.

\section{Other type material}

PORTUGAL • [12v and fragments], syntypes of Cardiomya cadiziana Huber, 2010 [= replacement name for Cardiomya curta (Jeffreys, 1876)]; off Algarve; 36 ${ }^{\circ} 54^{\prime} 00^{\prime \prime} \mathrm{N}, 8^{\circ} 14^{\prime} 30^{\prime \prime} \mathrm{W}$; 523-684 m depth; USNM 172032 • [5v], syntypes of Cardiomya cadiziana; São Pedro Canyon; 39 $55^{\circ} 00^{\prime \prime} \mathrm{N}, 9^{\circ} 56^{\prime} 00^{\prime \prime} \mathrm{W} ; 1818$ m depth; USNM 172033 • [4v], syntypes of Cardiomya cadiziana; São Pedro Canyon; 39² $42^{\prime} 00^{\prime \prime} \mathrm{N}$, 943'00" W; 1097-2003 m depth; USNM 172034.

SURINAME - [1v], holotype of Cardiomya surinamensis van Regteren Altena, 1971; 06 $22.5^{\prime} \mathrm{N}$, $55^{\circ} 10^{\prime} \mathrm{W} ; 20 \mathrm{~m}$ depth; RMNH.MOL.55387. 


\section{Description}

Shell small (length $4.3 \mathrm{~mm}$; height $3.2 \mathrm{~mm}$ ). Shell flank quadrangular, umbones raised. Anterodorsal margin forming a shoulder, anterior and ventral and margin rounded, little angulated. Rostrum very short, truncated, posteroventral sinuation shallow, posterodorsal margin curved upwards, extending

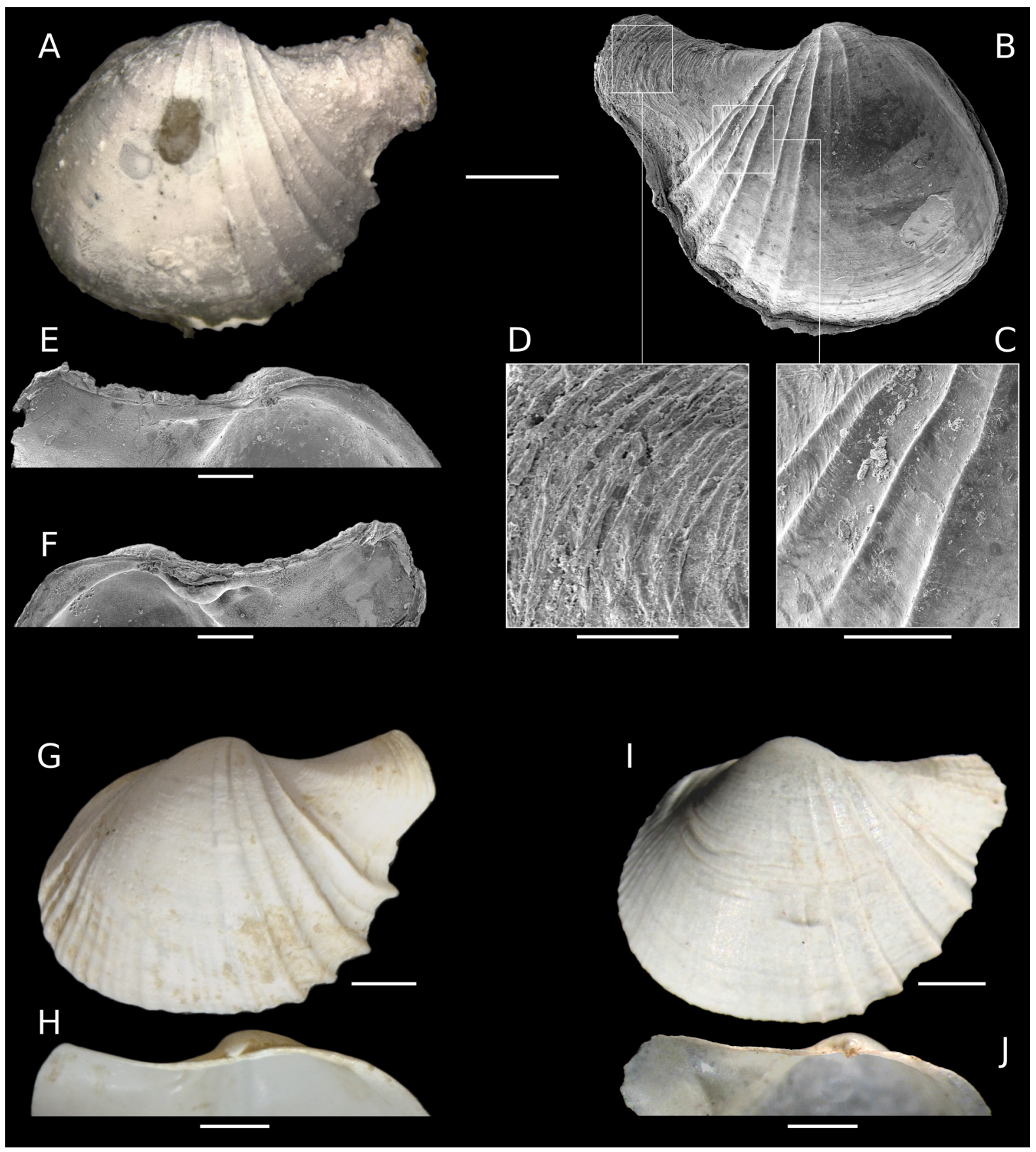

Fig. 5. A-F. Cardiomya minerva sp. nov., holotype (IBUFRJ 5785). A-B. External view, left and right valve. C-D. Details of the posterior radial ribs and the rostrum. E-F. Hinge detail. - G-H. Cardiomya surinamensis van Regteren Altena, 1971, holotype (RMNH.MOL.55387), left valve. G. External view. H. Hinge detail. - I-J. Cardiomya cadiziana Huber, 2010, syntype (USNM 172032), left valve. I. External view. J. Hinge detail. Scale bars: A-B, G-J $=1 \mathrm{~mm}$; $\mathrm{C}-\mathrm{D}=0.25 \mathrm{~mm}$; E-F $=0.5 \mathrm{~mm}$. 
above the umbones. External sculpture of six radial ribs on the posterior half of the shell flank; rostrum with commarginal growth lines only.

\section{Geographic distribution}

Only known from the type locality.

\section{Remarks}

The most similar species to Cardiomya minerva sp. nov. are Cardiomya surinamensis van Regteren Altena, 1971 (Fig. 5G-H) and Cardiomya cadiziana Huber, 2010 (Fig. 5I-J). These two species can be distinguished from Cardiomya minerva sp. nov. by their trapezoidal shell flank, external sculpture composed of incomplete radial ribs on anterior region of the shell flank. Additionally, C. cadiziana exhibits a shorter rostrum, with several faint radial lines, and straight posterodorsal margin, not extending above the umbones.

\section{Cardiomya sp. 1}

Fig. 6A-B

Cardiomya cleryana auct., non d'Orbigny, 1842 - Barroso et al. 2016: in part.

\section{Material examined}

BRAZIL - Amapá state • [1v]; northwest of Amazon Canyons; 03³7'00" S, 5001'00" W; $72 \mathrm{~m}$ depth; 08 May 1971; GEOMAR III, stn 193; CMPHRM 3681.

\section{Description}

Shell small (length $7.8 \mathrm{~mm}$; height $6.1 \mathrm{~mm}$ ). Shell flank elliptical, umbones blunt. Anterodorsal margin forming a shoulder, anterior margin truncated, ventral margin rounded, crenulated. Rostrum very short, truncated, posteroventral sinuation shallow, posterodorsal margin curved upwards, extending a little above the umbones. External sculpture of 22 radial ribs, less spaced towards the anterior region, the four strongest and most widely spaced ribs at the posterior end of the shell flank; rostrum with four radial lines.

\section{Geographic distribution}

Brazil, Amapá state, northwest of Amazon Canyons, $72 \mathrm{~m}$.

\section{Remarks}

Part of the specimens listed as Cardiomya cleryana in Barroso et al. (2016: 6) (CMPHRM 3681) are here reassigned to Cardiomya sp. 1.

Cardiomya surinamensis van Regteren Altena, 1971 is similar to Cardiomya sp. 1, but can be distinguished from it by its weak radial ribs, which vanish towards the anterior region, and the wide rostrum, devoid of radial ornamentation.

\section{Cardiomya sp. 2}

Fig. 6C-D

\section{Material examined}

BRAZIL - Amapá state • [1v]; off Salinópolis; 00²9'00" S, 47²4'00" W; 22 m depth; 15 Jun. 1971; GEOMAR III, stn 2533; CMPHRM 3668. 


\section{Description}

Shell small (length $5.8 \mathrm{~mm}$; height $4.1 \mathrm{~mm}$ ). Shell flank triangular, umbones blunt. Anterodorsal margin descending, anterior margin extended, ventral margin rounded, crenulated. Rostrum very short, posteroventral sinuation unobtrusive, posterodorsal margin slightly curved upwards, not extending much above the umbones. External sculpture of 17 radial ribs, many incomplete on the middle of the shell flank, followed by three strongest and most widely spaced complete radial ribs; rostrum with commarginal growth lines only.

\section{Geographic distribution}

Brazil, Amapá state, off Salinópolis, $22 \mathrm{~m}$.

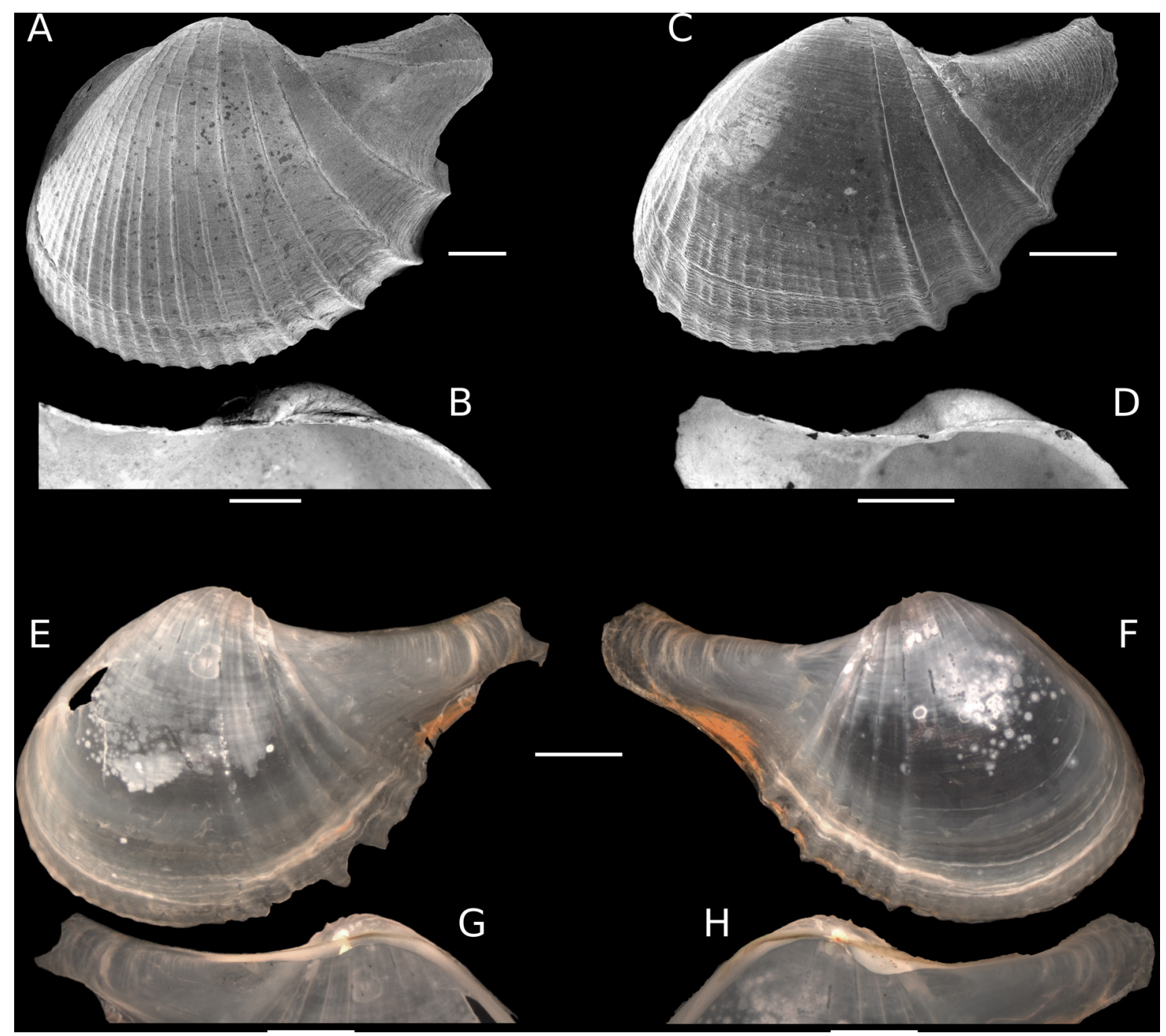

Fig. 6. A-B. Cardiomya sp. 1, CMPHRM 3681, left valve. A. External view. B. Hinge detail. C-D. Cardiomya sp. 2, CMPHRM 3668, left valve. C. External view. D. Hinge detail. - E-H. Cardiomya sp. 3, ZUEC-BIV 5128. E-F. External view, left and right valve. G-H. Hinge detail. Scale bars $=1 \mathrm{~mm}$. 
Cardiomya sp. 3

Fig. 6E-H

Cardiomya cleryana auct., non d'Orbigny, 1842 - Machado et al. 2016: in part.

\section{Material examined}

BRAZIL - São Paulo state • [1i]; São Sebastião, Biota Araçá; 2349'20.1" S, 45²4'10.3" W; 20 m depth; 21 Mar. 2014; ZUEC-BIV 5128.

\section{Description}

Shell small (length $6.2 \mathrm{~mm}$; height $4.2 \mathrm{~mm}$ ). Shell flank triangular, umbones blunt. Anterodorsal margin descending, anterior margin angulated, ventral margin rounded. Rostrum short, posteroventral sinuation shallow, posterodorsal margin slightly curved upwards, not extending much above the umbones. External sculpture of seven weak and incomplete radial ribs, which vanish towards ventral margin, followed by six complete ribs on the posterior half of the shell flank; rostrum with commarginal growth lines only.

\section{Geographic distribution}

Brazil, São Paulo state, off São Sebastião, 20 m.

\section{Remarks}

Part of the material identified by Machado et al. (2016) (ZUEC-BIV 5128) as C. cleryana, is here referred as Cardiomya sp. 3.

\section{Dichotomous key of the species from Brazilian waters}

1. Complete ribs covering the entire shell flank

- Complete ribs restricted to posterior half of the shell flank

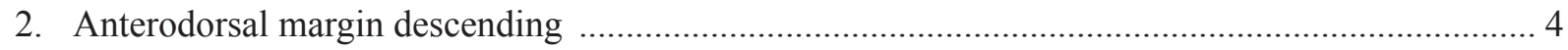

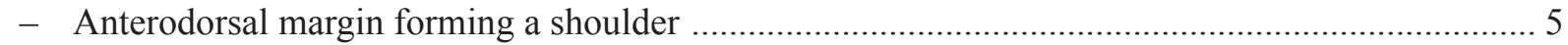

3. Rostrum short Cardiomya sp. 3

- Rostrum very short

4. Numerous ribs $(\sim 20)$; equidistant

Cardiomya cleryana (d'Orbigny, 1842)

- Few strong ribs $(\sim 6-12)$; very spaced; distributed throughout the shell flank

Cardiomya ornatissima (d'Orbigny, 1853)

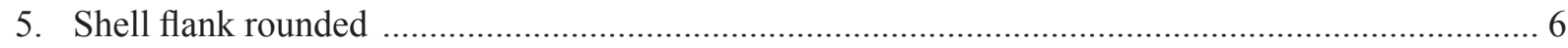

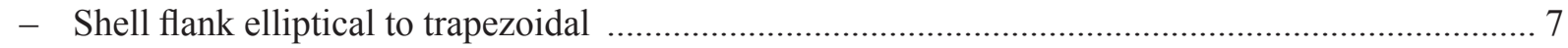

6. Rostrum long

- Rostrum very short Cardiomya sp. 1

7. Ribs $(\sim 30-50)$ equidistant, evenly distributed throughout the shell to the rostrum

- Ribs ( 18-35) more spaced in the posterior part of the shell flank.

Cardiomya striata (Jeffreys, 1876)

Cardiomya striolata (Locard, 1897)

8. Anterodorsal margin forming a shoulder Cardiomya minerva sp. nov.

- Anterodorsal margin descending Cardiomya sp. 2 


\section{Discussion}

The discovery of undescribed Cardiomya species hidden among the museum shelves in Brazilian collections reinforces the need for more taxonomic studies in this area. The combination of a reduced number of specialists, rarity of species and high cost of field expeditions hampers the improvement of our knowledge on marine biodiversity. It is not surprising that a large number of Cardiomya are still waiting proper description. But these limitations, especially the rarity of some species, should not prevent our attempts to understand and recognize them. Describing such species and their unique characteristics is the first step to understand their importance as components of natural ecosystems. It is worth noting that we do not comply with 'taxonomic splitting', which can lead to a rise in unwanted invalid names. We are aware that larger number of specimens allows for a more precise description of the full phenotypical variation for a species. Nevertheless, the cost of describing a rare species based on few specimens with noticeable diagnostic features seems lower than the cost of not knowing it at all. With predictions of massive extinctions in the near future, the next generation of taxonomists may be describing extinct species, only known from material preserved on the shelves (Fontaine et al. 2012).

\section{Acknowledgements}

We are grateful to the following for valuable exchange of information: Flávio Dias Passos, Fabrizio Marcondes Machado and Alan Rodrigo Batistão (all from UNICAMP); to Cristiane Xerez Barroso (LABOMAR-UFC); to Priscilla Grohmann (IBUFRJ) and Tito Cesar Marques de Almeida (UNIVALI). For providing information on lost types, we thank to Andreia Salvador and Tom White (both from NHMUK); to Emmanuel Robert (Musée de minéralogie MINES ParisTech); to José Leal (The BaileyMatthews National Shell Museum) and Virginie Heros (MNHN, Paris). For the photos of the types of C. striolata, we thanks to Phillippe Maestrati and Magalie Castelin (MNHN). For the figures of original illustration of $C$. costellata we thanks to Virginie Heros (MNHN). For their suggestions and critical review of this article we thank to André Sartori (Cambridge University); to Raquel Figueira (UFRJ); and to the anonymous reviewers. We also thank to all curators and staff of all institutions visited for their kind assistance and/or for arranging and shipping loans during the development of this study. During his participation in this study, the first author was financially supported by Programa Institucional de Bolsas de Iniciação Científica (PIBIC-UFRJ 2015-2017).

Funding. The first author was financially supported by "Programa Institucional de Bolsa de Iniciação Científica (PIBIC-UFRJ / EDITAL nº 84 CEG/CEPG 2016)".

Conflict of Interest. The authors declare that they have no conflict of interest.

Ethical approval. No animal testing was performed during this study.

Sampling and field studies. The study does not contain sampling material or data from field studies.

Data availability. All data generated or analysed during this study are included in this published article.

\section{References}

Abbott R.T. 1974. American Seashells. The Marine Molluska of the Atlantic and Pacific Coast of North America. $2^{\text {nd }}$ ed. Van Nostrand, New York.

Abbott R.T. \& Morris P.A. 1995. A Field Guide to Shells: Atlantic and Gulf Coasts and the West Indies. Houghton Mifflin Company, Boston / New York. 
Absalão R.S. \& Pimenta A.D. 2005. Moluscos Marinhos da APA do Arquipélago de Santana, Macaé, $R J$ - Chave para Identificação das Espécies do Substrato Inconsolidado. Editora Ciência Moderna, Rio de Janeiro.

Absalão R.S., Caetano C.H.S. \& Fortes F.R. 2006. Filo Mollusca. In: Lavrado H.P. \& Ignacio B.L. (eds), Biodiversidade Bentônica da Região Central da Zona Econômica Exclusiva Brasileira: 211-260. Serie Livros.Documentos REVIZEE/SCORE Central, Rio de Janeiro.

Allen J.A. 2011. Descriptions of new deep-water species of the family Cuspidariidae (Mollusca: Bivalvia) and including a bibliography of the known species from the Atlantic. Journal of Conchology 40 (4): $428-445$.

Allen J.A. \& Morgan R.E. 1981. The functional morphology of Atlantic deep water species of the families Cuspidariidae and Poromyidae (Bivalvia): an analysis of the evolution of the septibranch condition. Philosophical Transactions of the Royal Society of London, Series B 294: 413-546.

https://doi.org/10.1098/rstb.1981.0117

Ardovini R. \& Cossignani T. 2004. West African Seashells. L'Informatore Piceno, Ancona.

Barroso C.X., Salani S., Rabay S.G. \& Matthews-Cascon H. 2016. Septibranchia (Mollusca: Bivalvia) from the North and Northeast coasts of Brazil. Marine Biodiversity Records 9 (1): 1-14.

https://doi.org/10.1186/s41200-016-0004-y

Bouchet P. 2009. Cardiomya cleryana (d'Orbigny, 1842). MolluscaBase. World Register of Marine Species. Available from http://www.marinespecies.org/aphia.php?p=taxdetails\&id=408446 [accessed 13 Mar. 2019].

Castellanos Z.A. 1982. Los moluscos de las campañas del "Shinkai Maru". Neotropica 28 (79): 41-46.

Dall W.H. 1886. Reports on the results of dredging, under the supervision of Alexander Agassiz, in the Gulf of Mexico (1877-78) and in the Caribbean Sea (1879-80), by the U. S. Coast survey steamer "Blake", Lieut.-Commander C. D. Sigsbee, U. S. N., and Commander J. R. Bartlett, U. S. N., commanding. XXIX. Report on the Mollusca. Part 1. Brachiopoda and Pelecypoda. Bulletin of the Museum of Comparative Zoology 12 (6): 171-318.

Figueiras A. \& Sicardi O.E. 1970. Catálogo de los moluscos marinos del Uruguay. Comunicaciones de la Sociedad Malacológica del Uruguay 2 (18): 407-421.

Figueiras A. \& Sicardi O.E. 1980 [“1979”]. Catálogo de los moluscos marinos del Uruguay. Parte X. Revisión actualizada de los moluscos marinos del Uruguay con descripción de las especies agregadas. Sección I - Polyplacophora - Scaphopoda - Bivalvia. Comunicaciones de la Sociedad Malacológica del Uruguay 5: 107-157.

Fontaine B., Perrard A. \& Bouchet P. 2012. 21 years of shelf life between discovery and description of new species. Current Biology 22 (22): 943-944. https://doi.org/10.1016/j.cub.2012.10.029

Forcelli D.O. 2000. Moluscos Magallânicos: Guia de los Moluscos de la Patagonia y del Sur de Chile. Vazquez Mazzini, Buenos Aires.

Gofas S. 2015. Cardiomya (Adams, 1864). MolluscaBase. World Register of Marine Species. Available from http://www.marinespecies.org/aphia.php?p=taxdetails\&id=137857 [accessed 29 Mar. 2019].

Hinds R.B. 1843. Descriptions of new species of Necera, from the collection of Edward Belcher, C.B., made during a voyage round the world, and from that of Hugh Cuming, Esq., obtained during his visit to the Philippines; with notices of the synonymy. Proceedings of the Zoological Society of London 11: 75-79. 
Jeffreys J.G. 1865. British Conchology, or an Account of the Mollusca which now inhabit the British Isles and the surrounding Seas. Vol III. Marine Shells, comprising the remaining Conchifera, the Solenoconchia and Gasteropoda as far as Littorina.Van Voorst, London. https://doi.org/10.5962/bhl.title.16342

Jeffreys J.G. 1869. British Conchology, or an account of the Mollusca which now inhabit the British Isles and the surrounding Seas. Vol V. Marine Shells and naked Mollusca to the end of the Gastropoda, the Pteropoda, and Cephalopoda with a supplement and other matter, concluding the work. Van Voorst, London. https://doi.org/10.5962/bhl.title.16342

Lamy D. \& Pointier J.P. 2018. Marine and Freshwater Molluscs of the French Caribbean Vol II. PLB Editions, Guadeloupe.

Layerle C. \& Scarabino V. 1984. Moluscos del frente marítimo uruguayo entre 9 y $78 \mathrm{~m}$ de profundidad: análisis biocenológico. Contribuciones del Departamento de Oceanografía 1 (9): 1-17.

Lee H.G. 2009. Marine Shells of Northeast Florida. Jacksonville Shell Club, Florida.

Locard A. 1897. Notices conchyliologiques. XLVIII. Sur les Cuspidaria des côtes de France. L'Échange, Revue Linnéenne 13 (155): 94-95.

Locard A. 1898. Expéditions Scientifiques du Travailleur et du Talisman pendant les Années 1880, 1881, 1882, 1883. Mollusques Testacés vol. 2. G. Masson, Paris. https://doi.org/10.5962/bhl.title.98313

Locard A. 1899. Les Coquilles Marines au large des Côtes de France: Faune Pélagique et Faune Abyssale: Description des Familles, Genres et Espèces. Baillière, Paris.

https://doi.org/10.5962/bhl.title.14011

Machado F.M., Morton B. \& Passos F.D. 2016. Functional morphology of Cardiomya cleryana (d'Orbigny, 1842) (Bivalvia: Anomalodesmata: Cuspidariidae) from Brazilian waters: new insights into the lifestyle of carnivorous bivalves. Journal of the Marine Biological Association of the United Kingdom 97 (2): 447-462. https://doi.org/10.1017/S0025315416000564

Mikkelsen P.M. \& Bieler R. 2008. Seashells of Southern Florida: Living Marine Bivalves of the Florida Keys and Adjacent Regions. Princeton University Press, New Jersey.

Morretes F.L. 1949. Ensaio de catálogo dos moluscos do Brasil. Arquivos do Museu Paranaense, Curitiba 7: 5-216.

Morton B. 1981. The Anomalodesmata. Malacologia 21 (1-2): 35-60.

Morton B. 2016. The biology and functional morphology of the predatory septibranch Cardiomya costellata (Deshayes, 1833) (Bivalvia: Anomalodesmata: Cuspidariidae) from the Açores: survival at the edge. Journal of the Marine Biological Association of the United Kingdom 96 (6): 1347-1361. https://doi.org/10.1017/S0025315415001848

Oliver P.G., Holmes A.M., Killeen I.J. \& Turner J.A. 2016. Marine Bivalve Shells of the British Isles. Amgueddfa Cymru - National Museum Wales. Available from https://naturalhistory.museumwales.ac.uk/britishbivalves/ [accessed 25 Feb. 2019].

Oliveira C.D.C. \& Sartori A.F. 2014. Discovery and anatomy of the arenophilic system of cuspidariid clams (Bivalvia: Anomalodesmata). Journal of Morphology 275: 9-16.

https://doi.org/10.1002/jmor.20190

Oliveira C.D.C., Sartori A.F. \& Absalão R.S. 2017. Error cascade in taxonomy: the case of Cardiomya perrostrata (Mollusca: Bivalvia: Cuspidariidae) in Brazilian waters. Zootaxa 4247 (3): 281-300.

https://doi.org/10.11646/zootaxa.4247.3.3 
d'Orbigny A.D. 1842. Voyage dans l'Amérique Méridionale le Brésil, la République Orientale de l'Uruguay, la République Argentine, la Patagonie, la République du Chili, la République de Bolivia, la République du Pérou), exécuté pendant les années 1826, 1827, 1828, 1829, 1830, 1831, 1832 et 1833. Tome 5, Partie 3, Mollusques: I-XLIII, 1-758. P. Bertrand, Paris; V. Levrault, Strasbourg. Available from https://www.biodiversitylibrary.org/item/50739\#page/35/mode/1up [accessed 20 Mar. 2020].

d'Orbigny A.D. 1846. Voyage dans l'Amérique Méridionale (le Brésil, la République Orientale de l'Uruguay, la République Argentine, la Patagonie, la République du Chili, la République de Bolivia, la République du Pérou), exécuté pendant les années 1826, 1827, 1828, 1829, 1830, 1831, 1832 et 1833. Tome 9. Atlas zoologique (Mamifères, Oiseaux, Reptiles, Poissons, Mollusques, Polypiers, Foraminifères, Crustacés et Insectes). Atlas des Mollusques: pls 1-85. P. Bertrand, Paris; V. Levrault, Strasbourg. Available from https://www.biodiversitylibrary.org/item/50740\#page/7/mode/1up [accessed 20 Mar. 2020].

d'Orbigny A.D. 1853. Mollusques. Tome 2. In: de la Sagra R. (ed.) Histoire Physique, Politique et Naturelle de l'île de Cuba: 1-380. A. Bertrand, Paris.

Available from https://www.biodiversitylibrary.org/item/138987\#page/247/mode/1up [accessed 20 Mar. 2020].

Passos F.D. \& Magalhães F.T. 2011. A comparative study of the Bivalvia (Mollusca) from the continental shelves of Antarctica and Brazil. Biota Neotropica 11: 1-13.

https://doi.org/10.1590/S1676-06032011000100014

Philippi R.A. 1844. Enumeratio Molluscorum Siciliae cum viventium tum in tellure tertiaria fossilium, quae in itinere suo observavit Vol. 2. Schroppii, Berolini. https://doi.org/10.5962/bhl.title.100735

Porcheddu A.S., Ghisotti F. \& Castelli A. 1997. Ritrovamento di Cardiomya costellata (Deshayes, 1835) nelle acque antistanti l'Isola Tavolara (Sardegna nord-orientale) e discussione sulla validità specifica di Cardiomya striolata (Locard, 1898). Bollettino Malacologico 32 (5-8): 157-162.

Poutiers J.M. \& Bernard F.R. 1995. Carnivorous bivalve molluscs (Anomalodesmata) from the tropical western Pacific Ocean, with a proposed classification and a catalogue of recent species. In: Bouchet P. (ed.) Résultats des Campagnes MUSORSTOM 14. Mémoires du Muséum national d'Histoire naturelle 167: 107-187.

Powell A.W.B. 1951. Antarctic and subantarctic Mollusca: Pelecypoda and Gastropoda. Discovery Reports 26: 47-196. https://doi.org/10.5962/bhl.part.16335

Redfern C. 2001. Bahamian Seashells: a Thousand Species from Abaco, Bahamas. Boca Raton, Florida.

Rios E.C. 1970. Coastal Brazilian Seashells. Rio Grande, Rio Grande do Sul: Fundação Cidade do Rio Grande. Museu Oceanográfico de Rio Grande.

Rios E.C. 1972 [“1973”]. Moluscos marinos de la expedicion GEOMAR IV. Comunicaciones de la Sociedad Malacológica del Uruguay 3: 193-202.

Rios E.C. 1975. Brazilian Marine Mollusks Iconography. Fundação Universidade do Rio Grande, Centro de Ciências do Mar, Museu Oceanográfico, Rio Grande, Rio Grande do Sul.

Rios E.C. 1985. Seashells of Brazil. Fundação Cidade do Rio Grande. Fundação Universidade do Rio Grande. Museu Oceanográfico, Rio Grande, Rio Grande do Sul.

Rios E.C. 1994. Seashells of Brazil. Fundação Universidade do Rio Grande. Rio Grande.

Rios E.C. 2009. Compendium of Brazilian Sea Shells. Universidade Federal do Rio Grande, Museu Oceanográfico "Prof. E. C. Rios", Evangraf, Rio Grande. 
Salas C. 1996. Marine Bivalves from off the Southern Iberian Peninsula collected by the Balgim and Fauna 1 expeditions. Haliotis 25: 33-100.

Scarabino F. 2003a. Lista sistemática de los Bivalvia marinos y estuarinos vivientes de Uruguay. Comunicaciones de la Sociedad Malacológica del Uruguay 8 (80-81): 229-259.

Scarabino F. 2003b. Lista sistemática de los Cephalopoda vivientes de Uruguay. Comunicaciones de la Sociedad Malacológica del Uruguay 8 (78-79) 197-202.

Signorelli J.H., Fonseca M.L., Scarabino F. \& Passos F.D. 2019. The genus Dallocardia (Mollusca: Bivalvia: Cardiidae) in the Southwestern Atlantic Ocean. Marine Biodiversity 49: 2753-2773.

https://doi.org/10.1007/s12526-019-01004-3

Smith E.A. 1915. Mollusca. Part I. Gastropoda, Prosobranchia, Scaphopoda and Pelecypoda. British Antarctic ("Terra Nova") Expedition, 1910: Natural History Reports, Zoology 2 (4): 61-112.

Tunnell Jr J.W., Andrews J., Barrera N.C. \& Moretzsohn F. 2010. Encyclopedia of Texas Seashells: Identification, Ecology, Distribution, and History. Texas A\&M University Press.

Zelaya D.G. 2005. The bivalves from the Scotia Arc islands: species richness and faunistic affinities. Scientia Marina 69 (Suppl. 2): 113-122. https://doi.org/10.3989/scimar.2005.69s2113

Manuscript received: 6 June 2019

Manuscript accepted: 13 January 2020

Published on: 26 March 2020

Topic editor: Rudy Jocqué

Section editor: Thierry Backeljau

Desk editor: Pepe Fernández

Printed versions of all papers are also deposited in the libraries of the institutes that are members of the EJT consortium: Muséum national d'histoire naturelle, Paris, France; Meise Botanic Garden, Belgium; Royal Museum for Central Africa, Tervuren, Belgium; Royal Belgian Institute of Natural Sciences, Brussels, Belgium; Natural History Museum of Denmark, Copenhagen, Denmark; Naturalis Biodiversity Center, Leiden, the Netherlands; Museo Nacional de Ciencias Naturales-CSIC, Madrid, Spain; Real Jardín Botánico de Madrid CSIC, Spain; Zoological Research Museum Alexander Koenig, Bonn, Germany; National Museum, Prague, Czech Republic. 